\title{
ePsych: Interactive demonstrations and experiments in psychology
}

\author{
GARY L. BRADSHAW, BERNARD STEINMAN, and NANCY MCCARLEY \\ Mississippi State University, Mississippi State, Mississippi
}

\begin{abstract}
ePsych (http://epsych.msstate.edu), a new Web site currently under active development, is intended to teach students about the discipline of psychology. The site presumes little prior knowledge about the field and so may be used in introductory classes, but it incorporates sufficient depth of coverage to be useful in more advanced classes as well. Numerous interactive and dynamic elements are incorporated into various modules, orientations, and guidebooks. These elements include Java-based experiments and demonstrations, video clips, and animated diagrams. Rapid access to all material is provided through a layer-based navigation system that allows users to visit various "Worlds of the Mind." Active learning is encouraged, by challenging students with puzzles and problems and by providing the opportunity to "dig deeper" to learn more about the phenomena at hand.
\end{abstract}

ePsych (http://epsych.msstate.edu) is a developing Web site intended for students who are learning about psychology. ePsych follows in the footsteps of the Internet Psychology Lab (IPL) project (http://kahuna.psych.uiuc.edu/ipl), originated by Leonard J. Trejo, and incorporates several modules originally developed by the IPL team. As with other contemporary Web sites, such as CogLab (Francis et al., 2000), PsychExperiments (McGraw, Tew, \& Williams, 2000; Williams, McGraw, \& Tew, 1999), and the Cognitive Science Web site (Goolkasian \& van Wallendael, 2001), ePsych offers visitors a mix of on-line experiments and instruction. ePsych appears to be targeted at a more introductory level than are these other fine Web sites, making little or no assumptions about prior background in psychology. Unlike CogLab and PsychExperiments, ePsych is not intended as a primary source of laboratory experiments for students. Instead, the experiments included in ePsych play a more didactic role: They help students to appreciate the target concepts and ideas in a clearer light.

ePsych features numerous modules that provide short lessons on specific topics in psychology, whereas guidebooks and orientations provide a larger perspective. All of

This work was supported by Grants DUE 9981004 and DUE 0089420 from the National Science Foundation's Curriculum and Laboratory Improvement Program. In part, ePsych is based on the Internet Psychology Lab (IPL) sponsored by the University of Illinois. Site developers include the following: (IPL) Leonard J. Trejo, Michael Hamman, Chris Currie, Jordan McClure, Sheila Daniels, and Derek Kusiak; (ePsych) Chris Nolen, Clayton Graff, Connie Harris, and Beau Brady, with assistance from Jennifer Davidson, Brittny Mathies, and Kris Lee. The authors thank Sandra Goss-Lucas, Thomas Malloy, Jonathan Vaughn, and an anonymous reviewer for comments on the site and on the manuscript. Correspondence concerning this article should be addressed to G. L. Bradshaw, Department of Psychology, P. O. Box 6161, Mississippi State University, Mississippi State, MS 39762-6161(e-mail: glb2@ ra.msstate. edu). these units incorporate dynamic and interactive elements, such as Java-based experiments and demonstrations, QuickTime and Windows-Media video clips, and animated diagrams that illustrate various psychological principles, models, and phenomena.

To encourage active inquiry and exploration of the included material, ePsych exploits an exploration theme, "Journey into the Worlds of Mind." The main page (Figure 1) includes a "quantum flux teleporter" that transports visitors to different worlds, where they are met by guides at the visitor center. Maps of each world allow visitors to access individualmodules, orientations help visitors to understand the world, and guidebooks explain different areas within a world.

As an example, the "Descriptive Mind" world (which covers sensation, perception, and representation) has several islands (for vision, hearing, etc.). The orientation to this world relates the topics of sensation, perception, and representation to other aspects of human thinking (such as problem solving and communication). The guidebook "Secrets of Depth Valley" helps students to understand how three-dimensional vision arises from two-dimensional images. Some of the modules in this area include "Motion Parallax," "The Ball and Shadow Illusion," and "The Ponzo Illusion."

\section{ePsych Structure}

ePsych is based on a functional framework of the mind (Bradshaw, 1997). Currently, three different functional areas have been developed: the descriptive mind (sensation, perception, and representation), the deliberate mind (decision making, planning, and problem solving), and the adaptive mind (learning). New areas under development include the communicative mind (psycholinguistics), the executive mind (attention), and the biological mind (neuropsychology). 


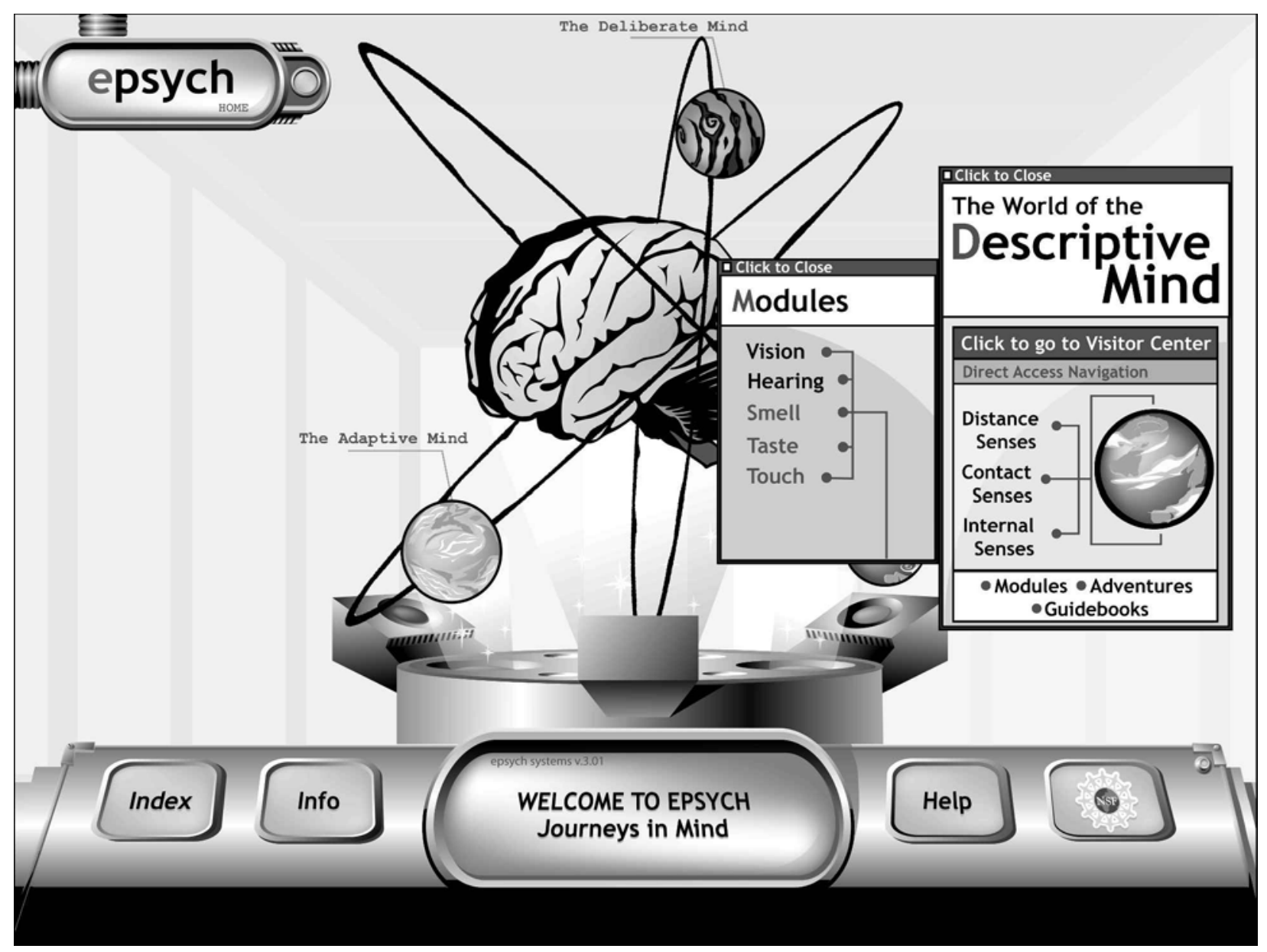

Figure 1. ePsych home page showing pop-up navigation layers for descriptive mind and distance senses.

Table 1 lists the contents of the site (as of March 2002) by the different functional areas.

The modules incorporate 780 different html files, 54 different Java applications, 2,329 image files, and 73 video clips, for a total of about 1.2 GBytes of on-line information. Featured video clips include films taken by Keith Hayes of the home-reared chimpanzee Viki (Hayes, 1962) and appear with his kind permission.

\section{ePsych Experiments}

As is shown in Table 1, ePsych includes a number of different experiments. Students act as subjects and are able to view their own data. Several different psychophysical methods are demonstrated in various modules. The Ponzo illusion experiment is based on the method of limits. The Poggendorf experiment is based on the method of constant stimuli. Müller-Lyer uses the method of adjustment. The horizontal/vertical experiment employs the method of adaptation. Each of these methods is described in some detail, allowing students to learn psychophysical methods as they perform experiments.

\section{Facilitating Ease of Use}

ePsych has several important features that facilitate user interaction. First, we have employed a consistent "lookand-feel" across the site, relying on a small set of templates to lay out Web pages. Template-based pages feature consistent navigation elements that provide straightforward linear movement through a module, as well as fast access to various indexes. Definitions of terms from the on-line glossary pop up in an overlay layer. The home page includes pop-up layer menus that allow direct access to the different worlds, regions, maps, guidebooks, orientations, and adventures. Finally, modules are typically written with a straightforward introductory section, followed by a branching tree that allows students to investigate variations that lead to a deeper appreciation of the material. These variations support a wide variety of users with different levels of experience and different goals.

\section{Fostering Active Learning}

ePsych incorporates a number of different elements to promote active learning on the part of students. First, mod- 
Table 1

ePsych Modules

\begin{tabular}{ll}
\hline \multicolumn{1}{c}{ Functional Area } & \multicolumn{1}{c}{ Element } \\
\hline The Descriptive Mind & \\
Vision & \\
Vision in Motion & Java demonstrations \\
Motion Parallax & Java demonstration \\
Ball and Shadow Illusion & Java demonstration \\
Apparent Motion & Java demonstration \\
Structure from Motion & Java demonstration \\
Biological Motion & Video demonstrations \\
Ponzo Illusion & Java demonstration and experiment \\
Poggendorf Illusion & Java demonstration and experiment \\
Ambiguous Figures & Animated demonstrations \\
Müller-Lyer Illusion & Java demonstration and experiment \\
Horizontal-Vertical Illusion & Java experiment \\
Change Blindness & Java demonstrations and experiment \\
Mental Rotation & Java experiment \\
Hearing & \\
Tone & Java demonstrations \\
Pitch & Java demonstrations and experiment \\
Shepard Tones & Java demonstrations \\
Deutsch Tritone Illusion & Java demonstrations \\
McGurk Effect & Video demonstrations \\
The Adaptive Mind & \\
Operant Conditioning & Java demonstration \\
Learning by Observation & Video demonstrations \\
Viki: A Cross-Fostered Chimpanzee & Video demonstrations \\
The Deliberate Mind & \\
The Stroop Effect & Java experiment \\
Signal Detection & Java experiment \\
\hline & \\
&
\end{tabular}

ules often begin with a puzzle that students must solve. Puzzles are not arbitrary but, instead, exemplify the issue at hand. This approach is designed to provoke curiosity about a topic. Next, interactive demonstrations allow students to explore the material. We have also adopted a friendly and casual style throughout the site. An on-line glossary is available to help students understand technical terms. We also will be implementing on-line quizzes in the near future that students can use to evaluate their mastery of the material. In some preliminary experiments, students have evaluated the presentation quite favorably in comparison with a traditional textbook coverage. Additional experiments are underway to measure the efficacy of the site as an educational tool.

\section{Conclusions}

ePsych complements traditional educational channels of classroom lectures and textbooks by providing students with a rich media experience of psychological topics. Students learn about various concepts by interacting with Javabased demonstrations, testing their own abilities in experiments, and watching video demonstrations of dynamic processes. Early tests of the site indicate that students ap- preciate the style and content of the site, which incorporates several mechanisms to promote easy access and active learning on the part of students.

\section{REFERENCES}

Bradshaw, G. L. (1997). Thought and language. In D. Bernstein (Ed.), Introduction to psychology (4th ed., pp. 263-301). Boston: Houghton Mifflin.

Francis, G., Neath, I., Stevenson, A. K., Surprenant, A. M., Kim, H., Kelley, M. R, \& Marble, J. (2000). CogLab Online Laboratory [On line]. Retrieved from http://coglab.psych.purdue.edu/coglab/index. html.

Goolkasian, P., \& VAn Wallendael, L. (2001). A web site in cognitive science. Behavior Research Methods, Instruments, \& Computers, 33, 258-262.

HAYES, K. (1962). Genes, drives, and intellect. Psychological Reports, 10, 299-342.

McGraw, K., Tew, M. D., \& Williams, J. E. (2000). Psych experiments: Psychology experiments on the Internet [On line]. Retrieved from http://www.olemiss.edu/projects/PsychExps.

Williams, J. E., McGraw, K. O., \& Tew, M. D. (1999). Undergraduate labs and computers: The case for PsychExps. Behavior Research Methods, Instruments, \& Computers, 31, 287-291.

(Manuscript received November 15, 2001; accepted for publication March 1, 2002.) 